Case Report

Open Access

CrossMark

\title{
SBRT (Stereotactic Body Radiation Therapy) - stereotactic body radiosurgery
}

\begin{abstract}
Stereotactic Body Radiation Therapy is a therapeutic modality to manage, in a few sessions and with great precision, very high doses of radiation to the tumor effectively and with minimal toxicity to surrounding healthy tissue. Side effects are few.
\end{abstract}

Keywords: radiation therapy, stereotactic body, toxicity, SBRT, precision, minimally invasive computed tomography, exactrac, brainlab, lung cancer, lumbar spine, pancreas, liver, cancer, tumors, metastasis
Volume 3 Issue 3 - 2015

Jasmina Alexander

Radiology and Radiation Therapy Technician, USA

Correspondence: Jasmina Alexander, Novalis Treatment Center, Panama City, Punta Pacifica John Hopkins Hospital, Basement 2, Panama, USA, Tel 507396 3162,

Email jasminaalexander@radiocirugiapanama.com

Received: October 0I, 20I5 | Published: October 30, 2015

\section{Case report}

Novalis powered by TrueBeam STx \& BrainLab ExacTrac

Female patient 60 years with 2 column injuries L1 and T12 treated each with 2 dynamic arches, 10 MV photons and 20 Gy FFF 120 MicroMLC HD in 5 sessions.

Stereotactic Body Radiation Therapy is a therapeutic modality to manage, in a few sessions and with great precision, very high doses of radiation to the tumor effectively and with minimal toxicity to surrounding healthy tissue. Side effects are few.

It is a treatment technique aimed at patients with tumors or metastases are inoperable and have injuries because of its location, can't be eradicated. The SBRT is a technique minimally invasive which allows the patient to receive treatment on an outpatient basis, without it is hospitalized.

The SBR requires precise planning to the target volume or target, since it is administered from different angles. This requires the use of 4 dimensional volumetric images mode, such as computed tomography (CT), taking into account the movement. These images are used to create individualized treatment plans that target the tumor accurately several radiation beams of different intensities and from different angles. The volumetric images are also taken immediately before treatment using imaging equipment directly connected to the computer for SBRT treatment (Figure 1). Another option is to implant a fiducial marker metal to track the position of the tumor with X-rays, or, placed on the markers patient fiducial with the same objective, which can be achieved by the help system ExacTrac of BrainLab, as shown in the pictures.

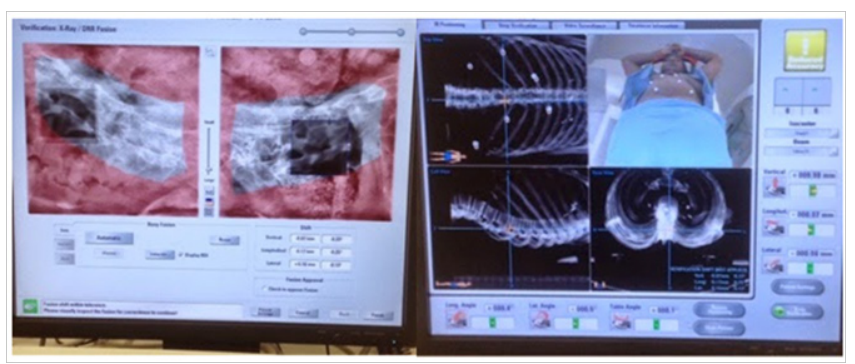

Figure I Volumetric images are taken immediately before treatment using imaging equipment directly connected to the computer for SBRT treatment.

Positioning system BrainLab ExacTrac of the First patient treated with SBRT in Panama with two thoracic injuries and lumbar spine respectively.

SBRT is used for treating lung cancer primarily and other cancers as the liver, pancreas and spine. Many cancers metastasize to the lung, and some initial results show that SBRT can improve local control of these metastases. 